\title{
The Clinical Value of Salivary Aldosterone in Diagnosis and Follow-Up of Primary Aldosteronism
}

\author{
Authors \\ U. D. Lichtenauer ${ }^{1,2}$, S. Gerum ${ }^{1,3}$, E. Asbach ${ }^{1}$, J. Manolopoulou ${ }^{1,4}$, V. Fourkiotis ${ }^{5,6}$, M. Quinkler ${ }^{5,7}$, \\ M. Bidlingmaier ${ }^{1}$, M. Reincke ${ }^{1}$ \\ Affiliations \\ Affiliation addresses are listed at the end of the article
}

\author{
Key words \\ - aldosterone \\ - rennin \\ - Conn's syndrome \\ - hypertension \\ - saliva \\ aldosterone producing \\ adenoma
}

received $\quad 02.03 .2016$

accepted 25.07.2016

\section{Bibliography}

DOI http://dx.doi.org/

10.1055/s-0042-114037

Published online:

September 2, 2016

Horm Metab Res 2016;

48: 638-643

(c) Georg Thieme Verlag KG

Stuttgart - New York

ISSN 0018-5043

\section{Correspondence}

\section{Reincke, MD}

Endocrinology, Medizinische Klinik und Poliklinik IV

Klinikum der Universität

München

Ziemssenstrasse 1

80336 Munich

Germany

Tel.: + 49/89/44005 2100

Fax: +49/89/44005 4428

martin.reincke@med.uni-

muenchen.de

\section{Abstract \\ $\nabla$}

Primary aldosteronism (PA), the most common form of secondary hypertension, causes relevant morbidity. The value of salivary measurements of aldosterone in clinical routine in PA so far has not been assessed. First, we analyzed salivary and plasma aldosterone concentrations of 42 patients with PA and 37 hypertensive controls (HC) during a sodium infusion test prospectively. Second, morning salivary and plasma aldosterone concentrations as well as diurnal saliva aldosterone profiles were analyzed in 115 patients treated for PA (46 adrenalectomy, 56 spironolactone, 13 eplerenone). Salivary aldosterone was substantially elevated in PA patients compared to $\mathrm{HC}$ at baseline ( $106 \pm 119$ vs. $40 \pm 21 \mathrm{ng} / \mathrm{l}, \mathrm{p}=0.01$ ), and after 4 -h sodium infusion test $(60 \pm 36$ vs. $23 \pm 14$, $\mathrm{p}=0.01$ ). Positive correlation between salivary

\section{Introduction}

$\nabla$

Hyperaldosteronism can be detected in $5-13 \%$ of hypertensive patients [1] and is associated with severe morbidity [2-4]. Aldosterone excess is caused primarily by aldosterone producing unilateral adenomas (APA) and bilateral adrenal hyperplasia (BAH) [5]. Most commonly, the diagnosis is established by an elevated plasma aldosteronerenin-ratio (ARR) and a pathologic confirmatory test such as the saline infusion test $[6,7]$. Unilateral adrenalectomy is the treatment of choice for aldosterone producing adenomas, while treatment with mineralocorticoid antagonists is recommended in bilateral disease $[8,9]$.

Repeated aldosterone measurements pre- and postoperatively and during follow-up visits are required for accurate monitoring of treatment effectiveness and outcome. Salivary aldosterone sampling has been proposed as a simple, costeffective and reliable method for these purposes and plasma aldosterone levels was evident, with exception of concentrations in or below the lower normal range. Applying a salivary aldosterone cutoff of $51.2 \mathrm{ng} / \mathrm{l}$, found by ROC curve analysis, rendered a sensitivity of $81 \%$ and a specificity of $73 \%$ for PA. The diurnal rhythm of aldosterone was preserved in untreated PA patients, but concentrations were higher in the context of PA, and normalized after surgery ( $118 \pm 57 \mathrm{vs.} 31 \pm 18 \mathrm{ng} / \mathrm{l}$, $\mathrm{p}<0.01)$. Taken together, salivary aldosterone measurements correlate with plasma levels, allowing simple and cost effective assessments of aldosterone secretion in an outpatient setting. Nevertheless, as this method alone cannot replace other plasma parameters, and as aldosterone profiling would not alter diagnostic or treatment strategies, salivary aldosterone measurements in routine practice are of limited clinical value.

[10-12]. As aldosterone is a lipid soluble steroid hormone, it readily passes through the cell membrane into saliva by simple diffusion. Salivary aldosterone concentrations are approximately $30 \%$ lower compared to plasma aldosterone concentrations, however, the diffusion rate is independent of saliva production [13] and salivary aldosterone levels correspond well with plasma aldosterone levels. Samples can easily be obtained without special training, and are stable for several days at room temperature [14]. We have previously shown that when measuring salivary aldosterone concentrations and applying a cutoff value of $81 \mathrm{pg} / \mathrm{ml}$, primary hyperaldosteronism was detected with a sensitivity of $77 \%$ and a specificity of $82 \%$ under experimental conditions [10].

In this study, aldosterone measurements from patients with primary hyperaldosteronism (PA) and hypertensive controls (HC) enrolled in the German Conn's Registry were prospectively per- 
formed in parallel in saliva and plasma during a saline infusion test. In addition, morning plasma aldosterone values taken at outpatient visits in our center were correlated with the patients' salivary aldosterone values taken at home, and the diurnal rhythm of aldosterone was assessed in the different cohorts. The effectiveness of salivary aldosterone daily profiling to monitor treatment outcomes after surgery and under mineralcorticoidantagonist treatment was addressed.

\section{Materials and Methods \\ $\nabla$ \\ Patients and test conditions}

The tertial referral centers LMU Munich and Charité Berlin had enrolled all patients in this study, approved by local ethic committees, by informed consent in the German Conn's Registry - Else Kröner-Fresenius-Hyperaldosteronismus-Registry. PA was diagnosed in accordance with the cutoff established in our laboratory and the Endocrine Society Practice Guidelines aldosterone concentration (ARR $>12 \mathrm{ng} / \mathrm{l} / \mu \mathrm{U} / \mathrm{ml}$ and aldosterone concentration $>50 \mathrm{ng} / \mathrm{l}$ after saline infusion) [7].

Seventy-nine patients with suspected PA were assessed by a saline infusion test prospectively. The diagnosis was confirmed in 42 and excluded in 37 patients. These 37 patients served as a hypertensive control (HC) cohort (demographic data $\odot$ Table 1). In addition, this test was performed again in 12 patients with persistent hypertension or elevated aldosterone-renin-ratios after adrenalectomy (demographic data $\bullet$ Table 2 ).

Furthermore, plasma and salivary aldosterone measurements in the morning as well as salivary aldosterone diurnal profiles were analyzed in 115 retrospective patients. These patients had been diagnosed with primary hyperaldosteronism months to years earlier, and were included in the registry during follow-up. The patients had been undergoing either adrenalectomy (ADX, $\mathrm{n}=46$ ) or mineralcocorticoid antagonist treatment in the case of $\mathrm{BAH}$ (spironolactone, $\mathrm{n}=56$, and eplerenone, $\mathrm{n}=13$; demographic data $\bullet$ Table 3). Patients were instructed to sample saliva at the time points 8:00, 12:00, 16:00, 20:00 and 22:00 h at home. All saliva samples were collected with the Salivette ${ }^{\circledR}$ (Sarstedt, Nümbrecht, Germany) device. Patients were asked not to eat or brush their teeth within 30 min before chewing the swab, and not to touch the swap. Besides these instructions, salivary samples were collected on an outpatient basis without further standardization. Samples were kept at $4{ }^{\circ} \mathrm{C}$ until transport to the hospital. Salivettes subsequently were centrifuged for $10 \mathrm{~min}$ at $2000 \mathrm{rpm}$ at $20^{\circ} \mathrm{C}$, and the flow-through collected for further analysis.

\section{Sodium infusion test}

The test was performed under standardized test conditions if primary aldosteronism was suspected as outlined in the Clinical Practice Guidelines [7]. Potassium was supplemented when the potassium concentration was below $3.5 \mathrm{mmol} / \mathrm{l}$ and interfering antihypertensive medication was changed to non-dihydropyridin calcium channel blockers and alpha-blockers whenever possible. Patient were fasting and seated for $10 \mathrm{~min}$, before 2 liters of saline solution $(0.9 \% \mathrm{NaCl})$ were infused over $4 \mathrm{~h}$ under blood pressure monitoring starting at 8:00 AM in the morning. At baseline and after $4 \mathrm{~h}$, plasma was drawn for measurements of aldosterone, renin, potassium, sodium, and creatinine. Salivary aldosterone samples were taken simultaneously. Contraindications were heart failure NYHA III-IV, a myocardial infarction within 3 months prior to testing, and hypertensive blood pressure readings ( $>180 \mathrm{mmHg}$ systolic, or $>100 \mathrm{mmHg}$ diastolic). Suppression of plasma aldosterone below $50 \mathrm{ng} / \mathrm{l}$ excluded a clinically relevant primary hyperaldosteronism.

Table 1 Demographic data of the prospective cohort and aldosterone concentrations in saliva and plasma of prospective cohort at baseline and after saline infusion (arithmetic mean, standard deviation, median), and correlation of plasma and salivary values.

\begin{tabular}{|c|c|c|c|c|}
\hline & Prospective cohort & & & \\
\hline & $\begin{array}{l}\text { Primary aldosteron- } \\
\text { ism }\end{array}$ & $\begin{array}{l}\text { Hyperten- } \\
\text { sive controls }\end{array}$ & After surgery & $\begin{array}{l}\text { Kruskal- } \\
\text { Wallis test }\end{array}$ \\
\hline Number of patients & 42 & 37 & 12 & \\
\hline Sex (male, female) & 31,11 & 14,23 & 9,3 & \\
\hline Age (years) & $53 \pm 10$ & $53 \pm 13$ & $61 \pm 11$ & \\
\hline Height (cm) & $175 \pm 8$ & $169 \pm 8$ & & \\
\hline Weight $(\mathrm{kg})$ & $87 \pm 19$ & $79 \pm 15$ & & \\
\hline Number of antihypertensives & $3 \pm 2$ & $2.5 \pm 2$ & 5/12 no antihypertensives & \\
\hline Systolic blood pressure (mmHg) & $164 \pm 28$ & $162 \pm 27$ & $150 \pm 17$ & \\
\hline Diastolic blood pressure $(\mathrm{mmHg})$ & $99 \pm 15$ & $97 \pm 17$ & $93 \pm 11$ & \\
\hline Serum potassium (mmol/l) & $3.1 \pm 0.5$ & $3.8 \pm 0.2$ & $4.1 \pm 0.2$ & \\
\hline Hypokalemic patients (\%) & $79(33 / 42)$ & $16(6 / 37)$ & & \\
\hline Aldosterone renin ratio & $109 \pm 159$ & $30 \pm 36$ & & \\
\hline Unilateral disease (\%) & $66(28 / 42)$ & & & \\
\hline Plasma aldosterone concentration at baseline (ng/I) & $288 \pm 180,227$ & $103 \pm 51,95$ & $71 \pm 33,72$ & $p<0.01$ \\
\hline Salivary aldosterone concentration at baseline (ng/l) & $106 \pm 119,74$ & $\begin{array}{l}40 \pm 21,41 \\
p \text { to } P A<0.01\end{array}$ & $\begin{array}{l}65 \pm 108,36 \\
p \text { to } H C=0.75\end{array}$ & $\mathrm{p}<0.01$ \\
\hline Correlation coefficient ( $r$ ) and $p$-value of correlation at baseline & $0.51,<0.01$ & $0.335,0.04$ & $-0.38,0.90$ & \\
\hline Plasma aldosterone concentration after sodium infusion (ng/l) & $181 \pm 141,115$ & $\begin{array}{l}35 \pm 1,35 \\
p \text { to } P A<0.01\end{array}$ & $\begin{array}{l}37 \pm 3,35 \\
\text { p to HC n.s. }\end{array}$ & $\mathrm{p}<0.001$ \\
\hline Salivary aldosterone concentration after sodium infusion (ng/l) & $60 \pm 36,51$ & $\begin{array}{l}23 \pm 14,20 \\
p \text { to } P A<0.01\end{array}$ & $\begin{array}{l}41 \pm 50,25 \\
p \text { to } H C=0.27\end{array}$ & $\mathrm{p}<0.001$ \\
\hline Correlation coefficient (r) and p-value of correlation after saline infusion & $0.6,<0.001$ & $-0.075,0.66$ & $0.4,0.188$ & \\
\hline
\end{tabular}

$r=$ Pearsons correlation coefficient; $p=$ significance level; $n$.s.: Not significant 
Table 2 Demographic data, aldosterone concentrations in saliva at different time points for daily profiling, and morning plasma aldosterone levels for comparison (arithmetic average, standard deviation) before and after surgery in paired samples.

\begin{tabular}{|llll} 
& \multicolumn{3}{c}{ Paired aldosterone profiles } \\
& Primary & After & Wilcoxon \\
& aldosteronism & surgery & test p \\
Number of patients & 12 & & \\
Sex (male, female) & 7,5 & & \\
Number of antihypertensives & $2 \pm 2$ & $0.9 \pm 1$ & \\
\hline Systolic blood pressure $(\mathrm{mmHg})$ & $154 \pm 21$ & $138 \pm 18$ & 0.046 \\
\hline Diastolic blood pressure $(\mathrm{mmHg})$ & $92 \pm 13$ & $88 \pm 16$ & 0.83 \\
\hline Serum potassium $(\mathrm{mmol} / \mathrm{l})$ & $3.3 \pm 0.6$ & $4.4 \pm 0.4$ & \\
\hline Aldosterone Renin Ratio & $182 \pm 218$ & $7 \pm 9$ & $<0.01$ \\
\hline Plasma aldosterone $(\mathrm{ng} / \mathrm{l})$ & $357 \pm 209$ & $76 \pm 43$ & $<0.01$ \\
\hline Salivary aldosterone $8: 00 \mathrm{~h}(\mathrm{ng} / \mathrm{l})$ & $118 \pm 57$ & $31 \pm 18$ & 0.01 \\
\hline Salivary aldosterone $12: 00 \mathrm{~h}(\mathrm{ng} / \mathrm{l})$ & $108 \pm 66$ & $31 \pm 29$ & $<0.01$ \\
\hline Salivary aldosterone $16: 00 \mathrm{~h}(\mathrm{ng} / \mathrm{l})$ & $86 \pm 61$ & $33 \pm 41$ & $<0.01$ \\
\hline Salivary aldosterone $20: 00 \mathrm{~h}(\mathrm{ng} / \mathrm{l})$ & $77 \pm 37$ & $21 \pm 14$ & $<0.01$ \\
\hline Salivary aldosterone $22: 00 \mathrm{~h}(\mathrm{ng} / \mathrm{l})$ & $70 \pm 36$ & $20 \pm 12$ & $<0.01$ \\
\hline
\end{tabular}

\section{Aldosterone measurements}

Salivary aldosterone was measured using a time-resolved fluorescence immunoassay (TR-FIA) as described previously [10]. Briefly, calibrators, controls and samples $(50 \mu \mathrm{l})$ were pipetted in duplicates, along with $100 \mu \mathrm{l}$ of aldosterone 3-CMO-biotin conjugate tracer in assay buffer, and incubated overnight at $4^{\circ} \mathrm{C}$. Streptavidin-Europium-antibody complexes were measured using a fluorometer (PerkinElmer LAS, Jügesheim, Germany) whereby concentrations of aldosterone present in the sample are inversely proportional to the signal generated. Aldosterone concentrations are reported in $\mathrm{ng} / \mathrm{l}$. The measurements in saliva were done over a period of several months, and aliquots from pooled samples were included as controls on each plate. All samples were measured in duplicates, and measurements were repeated if the coefficient of variability exceeded $20 \%$.

A commercially available aldosterone RIA (Siemens Medical Solutions Diagnostics, Bad Nauheim, Germanys) was used for measurement in plasma samples. The assay has a reported working range between $25-1200 \mathrm{ng} / \mathrm{l}$, analytical sensitivity of $11 \mathrm{ng} / \mathrm{l}$, and intra-/inter-assay CVs from 2.3-5.4\% and 3.8-15.7\%, respectively.

\section{Statistical analysis}

Quantitative values were depicted as statistical average and standard deviation. Statistical comparisons were performed using the Wilcoxon-Mann-Whitney test for 2 unmatched groups. More than 2 unmatched groups were compared using the nonparametric Kruskal-Wallis test. Linear dependencies between metric variables were quantified by applying Pearsons's correlations coefficient. 2 -sided p-values $<0.05$ were considered statistically significant. Due to the explorative nature of this project, a normalization of potential confounders was not performed.

\section{Results}

$\nabla$

\section{Salivary aldosterone at time of diagnosis and during} sodium infusion

Salivary aldosterone concentrations at 8:00 AM were significantly lower compared to plasma aldosterone concentrations and they differed significantly between patients with primary aldosteronism ( $\mathrm{PA} n=42$ ), hypertensive controls ( $\mathrm{HC}, \mathrm{n}=37)$, and after surgery $(\mathrm{n}=12)$ ( $\odot$ Table 1 and $\odot$ Fig. 1a). A significant correlation between salivary and plasma aldosterone levels was observed (PA r=0.51, p<0.01, O Fig. 1b; HC $r=0.335$, $\mathrm{p}=0.04 ; \odot$ Table 1). Applying a Receiver Operating Characteristic (ROC) curve analysis with a 95\% confidence interval for 8:00 AM salivary concentrations of PA vs. HC patients, an area under the curve (AUC) of $0.85(0.772-0.937$; std. error $0.04 ; \mathrm{p}<0.001)$ with a sensitivity of $81.0 \%(65.9-91.4)$ and a specificity of $73.0 \%$ (55.9-86.2) could be achieved at an aldosterone concentration of $51.15 \mathrm{ng} / \mathrm{l}(\bullet$ Fig. 1c).

Aldosterone concentrations after the saline infusion test differed significantly between PA and HC patients ( $\mathrm{p}<0.01)$, as expected, in both, saliva and plasma measurements. In contrast, salivary and plasma aldosterone concentrations in patients after adrenalectomy and HC patients were similar before $(p=0.75)$ and after $(p=0.27)$ saline infusion ( $\odot$ Table 1 and $\odot$ Fig. 1a).

Salivary and plasma aldosterone concentrations at 8:00 AM were analyzed in patients after successful adrenalectomy for PA and in BAH patients receiving spironolactone or eplerenone. Saliva and plasma aldosterone differed significantly between all groups while levels correlated mostly positively within each subgroup (newly diagnosed PA $r=0.719, p<0.01$; APA patients after surgery $r=0.285, p=0.058$; spironolactone treated $P A$ patients $\mathrm{r}=0.622, \mathrm{p}<0.01$; eplerenone treated $\mathrm{PA}$ patients $\mathrm{r}=0.817, \mathrm{p}<0.01 ; \odot$ Table 3 ).

Interestingly, subgroup analysis revealed that plasma aldosterone values at 8:00 AM were significantly higher in APA patients compared to BAH patients $(429 \pm 395$ vs. $184 \pm 73 \mathrm{ng} / \mathrm{l}, \mathrm{p}=0.013)$. Although this tendency was also seen for salivary aldosterone, a statistical significance was not reached (APA $n=29,120 \pm 140 \mathrm{ng} / \mathrm{l}$ vs. $\mathrm{BAH} \mathrm{n}=9,76 \pm 25 \mathrm{ng} / \mathrm{l}, \mathrm{p}=0.36$; data not shown).

\section{Salivary aldosterone profiling}

In untreated patients with $\mathrm{PA}$, higher aldosterone levels (PA $\mathrm{n}=42 ; 100 \pm 73 \mathrm{ng} / \mathrm{l}$ vs. APA patients after successful adrenalectomy $\mathrm{n}=48 ; 59 \pm 43 \mathrm{ng} / \mathrm{l}, \mathrm{p}<0.05)$ and a steeper decline within $24 \mathrm{~h}$ was observed ( $\odot$ Fig. 1d). In contrast, aldosterone curves of spironolactone treated BAH patients $(n=56,11$ spironolactone alone, 45 combinatory therapy; average dose: $60 \pm 41$ (13-200) $\mathrm{mg} /$ day) and patients with untreated PA, with the exception of 22:00 ( $p=0.01$ ), were similar ( $\bullet$ Table 3 and $\odot$ Fig. 1 d). Salivary aldosterone concentrations also tended to be higher in eplerone treated patients $(\mathrm{n}=13,7$ eplerenone alone, 6 combinatory therapy, average dose $74 \pm 45(13-150) \mathrm{mg} /$ day $)$ compared to untreated patients, and statistical significance was reached at 16:00 $(\mathrm{p}=0.04)$ and 22:00 $\mathrm{h}(\mathrm{p}<0.01)$.

In 12 patients, who remained hypertensive or still had an elevated aldosterone-renin-ratio after surgery, paired pre- and postoperative salivary aldosterone profiles were available (๑ Table 2). Again, a maintained diurnal rhythm on a much higher level was observed for patients before surgery. Both, salivary and plasma aldosterone concentrations after surgery were much lower at 8:00 AM in the morning and at all other time points studied with salivary profiling (8:00 AM salivary: $118 \pm 57 \mathrm{ng} / \mathrm{l}$ before and $31 \pm 18 \mathrm{ng} / \mathrm{l}$ after surgery, $\mathrm{p}<0.01$; $\odot$ Fig. 1e; plasma $345 \pm 204 \mathrm{ng} / \mathrm{l}$ before and $73 \pm 43 \mathrm{ng} / \mathrm{l}$ after surgery, $\mathrm{p}<0.01$; paired values $\odot$ Fig. 1f). 
Table 3 Demographic data of retrospective cohort.

Number of patients

Sex (male, female)

Age (years)

Number of antihypertensives

Dose $(\mathrm{mg} / \mathrm{d})$

Systolic blood pressure $(\mathrm{mmHg})$

Diastolic blood pressure $(\mathrm{mmHg})$

Serum potassium ( $\mathrm{mmol} / \mathrm{l})$

Plasma aldosterone 8:00 h (ng/l)

Salivary aldosterone 8:00 h (ng/l)

p-Value to untreated primary aldosteronism $8: 00 \mathrm{~h}$

Correlation coefficient $(r)$ and $p$-value of correlation

Salivary aldosterone 12:00 h (ng/l)

p-Value to untreated primary aldosteronism 12:00 h

Salivary aldosterone 16:00 h (ng/l)

p-Value to untreated primary aldosteronism 12:00 h

Salivary aldosterone 20:00 h (ng/l)

p-Value to untreated primary aldosteronism 20:00 h

Salivary aldosterone 22:00 h (ng/l)

$\mathrm{p}$-Value to untreated primary aldosteronism 22:00 h

Aldosterone concentrations in saliva at different time points for daily profiling and morning plasma aldosterone levels for comparison (arithmetic mean, standard deviation) Aldosterone concentrations in saliva at different time points for daily profiling and morning plasma aldosterone levels for comparison (arithmetic mean, standard deviation)

Correlation of plasma and salivary values: $r=$ Pearsons correlation coefficient; $p=$ significance level

\section{Discussion}

A systematic screening for clinically relevant hyperaldosteronism warrants repeated aldosterone measurements, both, for establishing the diagnosis and reliable follow-up. Determination of aldosterone levels based on a fluorescence-immunoassay in saliva has been proposed as a simple and cost effective alternative in previous studies [10]. In this study, the suitability and validity of this so far experimental method was assessed in a large patient cohort under routine clinical conditions. In some, but not all of our samples, a close correlation between salivary and plasma aldosterone levels could be found. At 8:00 AM a sensitivity of $81 \%$ and a specificity of $73 \%$ for detecting hyperaldosteronism utilizing saliva samples could be achieved, in agreement to what we have previously published, however, at a much lower cutoff level of $51.2 \mathrm{ng} / \mathrm{l}$, which is most likely attributed to the different cohorts studied. However, although sensitivity and specificity are in the range reported for other diagnostic tools, this method alone, similar to plasma testing alone, is not suitable as a screening method to reliably detect patients with pathologically elevated aldosterone concentrations.

In addition, as saliva contains the free, non-protein bound hormone, salivary aldosterone concentrations are generally much lower compared to plasma levels. Differences between the patient cohorts studied with values in the lower normal range, for example, after the saline infusion test, became minimal and did not reach statistical significance. This lack of discriminatory power at lower levels, however, is not deemed clinically relevant in most cases.

While plasma aldosterone levels were significantly higher in unilateral disease compared to bilateral disease, these differences could not be seen with salivary aldosterone measurements, most likely due to the larger scatter of values in the APA group.
Salivary hormone assays allow simple multiple non-invasive aldosterone measurements within an outpatient setting. Herein, the circardian rhythm of salivary aldosterone was assessed on an outpatient basis on a large cohort for the first time, and the results are similar to plasma measurements in primary hyperaldosteronism published earlier $[15,16]$. The profiles mirror well the typical diurnal rhythm of plasma aldosterone, with the highest concentrations in the morning hours, which gradually decline throughout the day. Interestingly, a disruption of the diurnal rhythm, for example, similar to patients suffering from hypercortisolism, could not be observed in PA. We can only speculate that the differences in aldosterone concentrations between hypertensive controls and APA patients were not severe enough to completely suppress the circardian rhythm. However, the decline of aldosterone concentrations throughout the day is much less pronounced in the APA group, suggesting that the autonomous aldosterone secretion in this group might have precluded a further decline. However, aldosterone levels were significantly higher compared to aldosterone profiles in hypertensive controls, and surgical intervention resulted in a normalization of the salivary aldosterone profile. Interestingly, this was also seen in the majority of patients, who remained hypertensive or with elevated aldosterone-renin-ratios after surgery. This emphasizes the difficulty to adequately define a successful treatment.

Plasma and salivary aldosterone measurements did not always correlate significantly in these subgroups, but there was a general trend for higher salivary aldosterone concentrations in patients under aldosterone antagonist treatment compared to untreated patients. Salivary aldosterone concentrations were highest in the eplerenone treated group. Several reason might explain this difference between groups, such as a per se higher disease activity in eplerenone treated patients, lower medical adherence in spironolactone treated patients, or differences in 

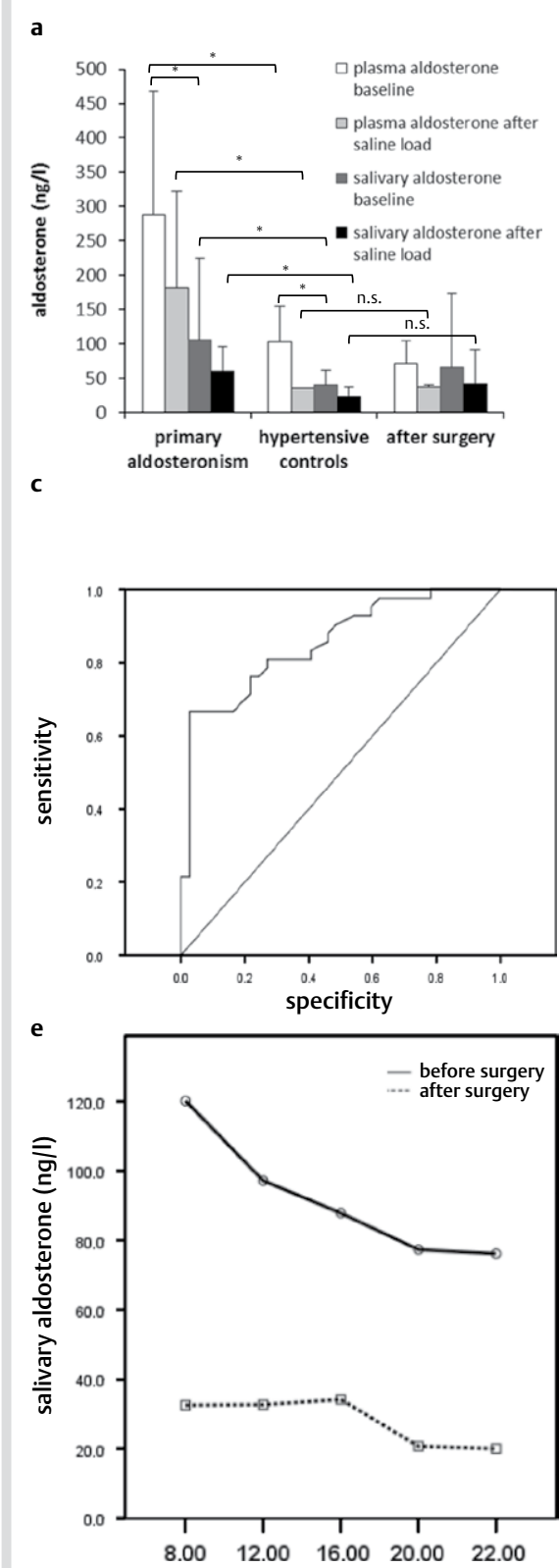

b

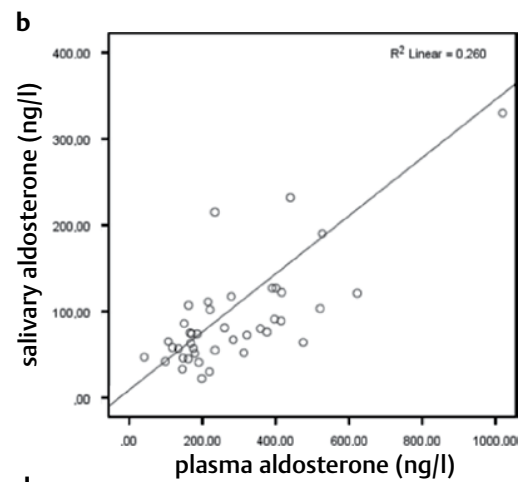

d

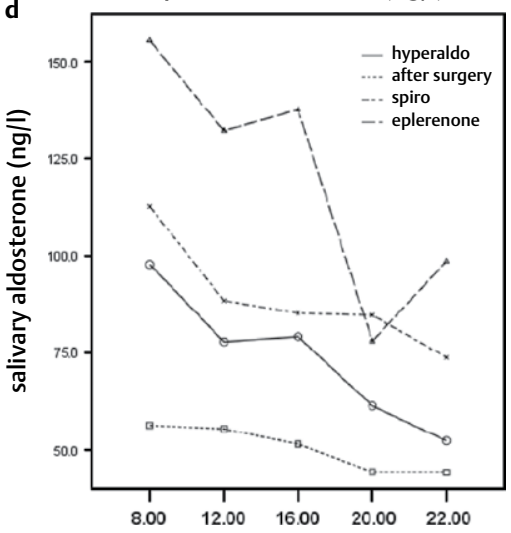

f

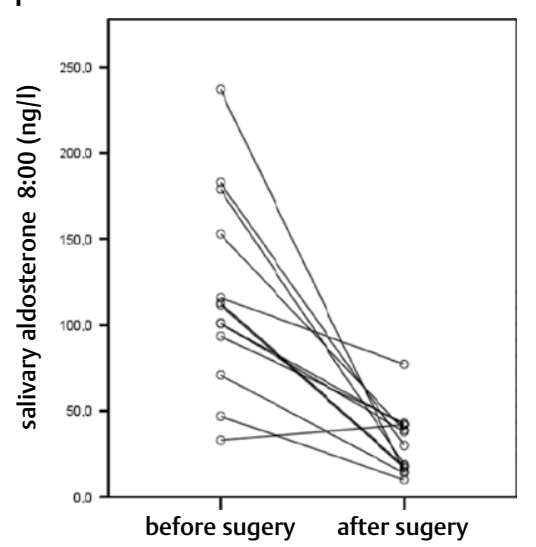

Fig. 1 Aldosterone concentrations in saliva and plasma of prospective cohort at baseline and after saline infusion depicted as a comparative bar graph a. Correlation of plasma and salivary values as a linear regression plot exemplarily depicted for untreated patients with hyperaldosteronism b. ROC curve for identifying patients with primary aldosteronism with salivary aldosterone measurements at 8:00 AM c. Mean salivary aldosterone concentrations of the different subgroups shown at different time points throughout the day in a comparative dot plot $\mathbf{d}$. Mean salivary aldosterone concentrations pre- and postoperatively shown at different time points throughout the day in a comparative dot plot e. Individual changes in salivary aldosterone concentrations before and after surgery $\mathbf{f}$. pharmacokinetic profile or mineralocorticoid receptor affinity. The remarkable increase of aldosterone concentrations after 12:00 and 20:00 h might reflect the pharmacological action of eplerenone, which was given twice daily. However, statistical significance was not reached for several times points, most likely due to the small cohort size of the subpopulations. Therefore, although these different aldosterone profiles are interesting, clearly the data does not allow to test aldosterone antagonist treatment efficiency or dose titration by salivary aldosterone sampling.

Taken together, aldosterone profiling with multiple saliva measurements a day is a simple, non-invasive method. The lack of standardization, the lack of a closer correlation with plasma aldosterone measurements, and lack of relevant additional information from daily profiles, together with the need for further laboratory parameters for proper clinical testing and follow-up, substantiates, that this method is of little clinical value in its current form.
Strength and limitations of the study: A strength of this study is that comparable plasma and salivary measurements were taken on a large patient cohort under standardized conditions set by the German Conn Registry. Another, that parts of the data were collected prospectively, allowing accurate sensitivity and specificity calculations. In addition, paired aldosterone measurements pre- and postoperatively in 12 patients allow to demonstrate treatment efficacy, even when hyperkalemia or hypertension persists. The results are limited by the fact that the number of patients is still not large enough to reliably discriminate aldosterone concentration differences at lower concentrations and in different subgroups. Limitations of the study include the sensitivity of the aldosterone assays, making reproducibility and agreement at low aldosterone concentrations questionable. Such limitations have been reported for plasma aldosterone concentrations before [17], but might be even more important for salivary aldosterone concentrations, which are even lower than those found in plasma. 


\section{Acknowledgements}

This work was funded by a grant of the Else Kröner-Fresenius Stiftung in support of the German Conn's Registry-Else KrönerFresenius-Hyperaldosteronism Registry (2013_A182).

\section{Conflicts of Interest}

\section{$\nabla$}

The authors declare no conflict of interest.

\section{Affiliations}

${ }^{1}$ Department of Endocrinology, Medizinische Klinik und Poliklinik IV, Klinikum der Universität München, Munich, Germany

${ }^{2}$ Department of General Internal Medicine, Endocrinology and Diabetology, HELIOS Kliniken, Schwerin, Germany

${ }^{3}$ Klinik und Poliklinik für Strahlentherapie und Radioonkologie, Klinikum der Universität München, Munich, Germany

${ }^{4}$ Immunodiagnostic Systems Ltd, Boldon, Tyne and Wear, U. K.

${ }^{5}$ Clinical Endocrinology, Campus Mitte, Charité University Medicine Berlin, Berlin, Germany

${ }^{6}$ Department of Gastroenterology and Endocrinology, Philipp's University,

Marburg, Germany

${ }^{7}$ Endocrinology in Charlottenburg, Berlin, Germany

\section{References}

1 Hannemann A, Bidlingmaier M, Friedrich N, Manolopoulou J, Spyroglou A, Volzke H, Beuschlein F, Seissler J, Rettig R, Felix SB, Biffar R, Doring A, Meisinger C, Peters A, Wichmann HE, Nauck $M$, Wallaschofski $H$, Reincke $M$. Screening for primary aldosteronism in hypertensive subjects: results from two German epidemiological studies. Eur J Endocrinol 2012; 167: 7-15

2 Milliez P, Girerd X, Plouin PF, Blacher J, Safar ME, Mourad JJ. Evidence for an increased rate of cardiovascular events in patients with primary aldosteronism. J Am Coll Cardiol 2005; 45: 1243-1248

3 Quinkler M, Born-Frontsberg E, Fourkiotis VG. Comorbidities in primary aldosteronism. Horm Metab Res 2010; 42: 429-434

4 Reincke M, Fischer E, Gerum S, Merkle K, Schulz S, Pallauf A, Quinkler M, Hanslik G, Lang K, Hahner S, Allolio B, Meisinger C, Holle R, Beuschlein F, Bidlingmaier M, Endres S.German Conn's Registry-Else Kroner-Fresenius-Hyperaldosteronism $R$. Observational study mortality in treated primary aldosteronism: the German Conn's registry. Hypertension 2012; 60: 618-624
5 Zennaro MC, Fernandes-Rosa F, Boulkroun S, Jeunemaitre X. Bilateral Idiopathic Adrenal Hyperplasia: Genetics and Beyond. Horm Metab Res 2015; 47: 947-952

6 Rossi GP, Dalla CA, Italian Society of $H$. Clinical management of primary aldosteronism: 2013 Practical Recommendations of the Italian Society of Hypertension (SIIA). High Blood Press Cardiovasc Prev 2014; 21: 71-75

7 Funder JW, Carey RM, Fardella C, Gomez-Sanchez CE, Mantero F, Stowasser M, Young WF Jr., Montori VM. Endocrine S. Case detection, diagnosis, and treatment of patients with primary aldosteronism: an endocrine society clinical practice guideline. J Clin Endocrinol Metab 2008; 93: 3266-3281

8 Riester A, Reincke $M$, Beuschlein F. Primary hyperaldosteronism: boundaries of diagnostics and therapy. Dtsch Med Wochenschr 2014; 139: 1982-1984

9 Quinkler M, Stewart PM. Treatment of primary aldosteronism. Best Pract Res Clin Endocrinol Metab 2010; 24: 923-932

10 Manolopoulou J, Gerum S, Mulatero P, Rossignol P, Plouin PF, Reincke $M$, Bidlingmaier $M$. Salivary aldosterone as a diagnostic aid in primary aldosteronism. Horm Metab Res 2010; 42: 400-405

11 Manolopoulou J, Mulatero P, Maser-Gluth C, Rossignol P, Spyroglou A, Vakrilova Y, Petersenn S, Zwermann O, Plouin PF, Reincke M, Bidlingmaier $M$. Saliva as a medium for aldosterone measurement in repeated sampling studies. Steroids 2009; 74: 853-858

12 Atherden SM, Corrie JE, Jones DB, Al-Dujaili EA, Edwards CR. Development and application of a direct radioimmunoassay for aldosterone in saliva. Steroids 1985; 46: 845-855

13 Lewis JG. Steroid analysis in saliva: an overview. Clin Biochem Rev 2006; 27: 139-146

14 Hofman LF. Human saliva as a diagnostic specimen. J Nutr 2001; 131 : 1621S-1625S

15 Kem DC, Weinberger MH, Gomez-Sanchez C, Kramer NJ, Lerman $R$, Furuyama $S$, Nugent $C A$. Circadian rhythm of plasma aldosterone concentration in patients with primary aldosteronism. J Clin Invest 1973; 52: 2272-2277

16 Sonoyama T, Sone M, Tamura N, Honda K, Taura D, Kojima K, Fukuda Y, Kanamoto N, Miura M, Yasoda A, Arai $H$, Itoh $H$, Nakao K. Role of endogenous ACTH on circadian aldosterone rhythm in patients with primary aldosteronism. Endocr Connect 2014; 3: 173-179

17 Schirpenbach C, Seiler L, Maser-Gluth C, Beuschlein F, Reincke M, Bidlingmaier M. Automated chemiluminescence-immunoassay for aldosterone during dynamic testing: comparison to radioimmunoassays with and without extraction steps. Clin Chem 2006; 52: 1749-1755 